\title{
Corticospinal output and loss of force during motor fatigue
}

\author{
Kai M. Rösler $\cdot$ O. Scheidegger $\cdot$ M. R. Magistris
}

Received: 8 August 2008 / Accepted: 5 June 2009 / Published online: 2 July 2009

(C) Springer-Verlag 2009

\begin{abstract}
The objective of this study was to analyze central motor output changes in relation to contraction force during motor fatigue. The triple stimulation technique (TST, Magistris et al. in Brain 121(Pt 3):437-450, 1998) was used to quantify a central conduction index $(\mathrm{CCI}=$ amplitude ratio of central conduction response and peripheral nerve response, obtained simultaneously by the TST). The CCI removes effects of peripheral fatigue from the quantification. It allows a quantification of the percentage of the entire target muscle motor unit pool driven to discharge by a transcranial magnetic stimulus. Subjects $(n=23)$ performed repetitive maximal voluntary contractions (MVC) of abductor digiti minimi (duration $1 \mathrm{~s}$, frequency $0.5 \mathrm{~Hz}$ ) during $2 \mathrm{~min}$. TST recordings were obtained every $15 \mathrm{~s}$, using stimulation intensities sufficient to stimulate all cortical motor neurons (MNs) leading to the target muscle, and during voluntary contractions of $20 \%$ of the MVC to facilitate the responses. TST was also repetitively recorded during recovery. This basic exercise protocol was modified in a number of experiments to further characterize influences on CCI of motor fatigue (4 min exercise at 50\% MVC; delayed fatigue recovery during local hemostasis, "stimulated exercise" by $20 \mathrm{~Hz}$ trains of $1 \mathrm{~s}$ duration at $0.5 \mathrm{~Hz}$ during $2 \mathrm{~min}$ ). In addition, the corti-
\end{abstract}

K. M. Rösler $(\bowtie) \cdot$ O. Scheidegger

Department of Neurology, Inselspital,

Bern University Hospital, University of Bern,

3010 Bern, Switzerland

e-mail: kai-michael.roesler@insel.ch

M. R. Magistris

Department of Neurology,

Hôpital Cantonal de Genève, Geneva University,

Geneva, Switzerland cal silent period was measured during the basic exercise protocol. Force fatigued to approximately $40 \%$ of MVC in all experiments and in all subjects. In all subjects, CCI decreased during exercise, but this decrease varied markedly between subjects. On average, CCI reductions preceded force reductions during exercise, and CCI recovery preceded force recovery. Exercising at $50 \%$ for $4 \mathrm{~min}$ reduced muscle force more markedly than CCI. Hemostasis induced by a cuff delayed muscle force recovery, but not CCI recovery. Stimulated exercise reduced force markedly, but CCI decreased only marginally. Summarized, force reduction and reduction of the CCI related poorly quantitatively and in time, and voluntary drive was particularly critical to reduce the CCI. The fatigue induced reduction of CCI may result from a central inhibitory phenomenon. Voluntary muscle activation is critical for the CCI reduction, suggesting a primarily supraspinal mechanism.

Keywords Triple stimulation technique - Transcranial magnetic stimulation $\cdot$ Central motor conduction $\cdot$ Motor evoked potential $\cdot$ Maximal voluntary force $\cdot$ Fatigue

\section{Introduction}

Motor fatigue is defined as an exercise-induced decrease of the maximal muscular force (Edwards et al. 1977). A number of studies have shown that motor fatigue is associated with alterations of motor conduction within the central nervous system (CNS), since motor evoked potentials (MEPs) to transcranial magnetic brain stimulation (TMS) can be altered after fatiguing muscular contractions (Brasil-Neto et al. 1993; McKay et al. 1995; Liepert et al. 1996; Samii et al. 1997; Gandevia et al. 1999; Pitcher and Miles 2002; Taylor et al. 1996, 1999). Andersen et al. (2003) applied 
the triple stimulation technique (TST; see below) to quantify the number of activated motor neurons during fatigue. They demonstrated marked reductions of motor neuron activation by TMS, suggesting alterations in cortical function and loss of force-producing ability. Theoretically, if reduced central motor output caused the loss of muscular force during fatigue, then loss of force and reduction of central conduction should parallel each other. One of the goals of the present study was therefore to investigate the quantitative relation and the time course of muscle force and central conduction during motor fatigue. A second purpose of the study was to investigate the role of voluntary drive on the behavior of the TMS evoked responses, by comparing fatigue induced by voluntary exercise with that elicited by electrically induced muscle contractions (Pitcher and Miles 2002).

Quantification of central conduction is not trivial, and conventional MEPs are not reliable to measure changes in central conduction (Rösler et al. 1999; Humm et al. 2004). A number of difficulties arise when using TMS to assess central outflow during fatigue:

- First, during fatigue, peripheral changes occur, affecting the size and shape of the compound muscle action potential (CMAP) obtained by peripheral nerve stimulation (McManis et al. 1986). Any changes in central outflow are thus superimposed on these peripheral changes. This effect is difficult to control when using conventional TMS, since central (TMS) and peripheral stimuli cannot be given simultaneously.

- Second, if a stimulus is given during a contraction of the target muscle, the size of the MEP will be facilitated, by spinal or cerebral mechanisms, or both. The relation between the degree of muscular background contraction and facilitation is not linear, and differs between muscles (Hess et al. 1987; Kischka et al. 1993; Arányi et al. 1998). Thus to control for this facilitation, the amount of muscular contraction must be kept constant, which is problematic when the force generating capacity of a muscle changes during fatigue. Most previous TMS fatigue studies relied therefore on stimuli given either at rest or during maximal contractions. While stimuli at rest may fail to recruit a large proportion of the motor neuron pool supplying the target muscle, stimuli during maximal contractions often yield distorted responses due to the ongoing muscle activity, which are difficult to assess.

- Third, the size of MEPs is not directly related to the number of TMS-induced motor neuron discharges, since these discharges are desynchronized. This desynchronization changes from one brain stimulus to the next (Magistris et al. 1998), and differs substantially (and unpredictably) between subjects (Rösler et al. 2002). Discharge desynchronization reduces the size of MEPs, and variation of desynchronization causes MEPs to vary in size from one stimulus to the next (Magistris et al. 1998; Rösler et al. 2002). It is known that during voluntary activation, muscle fatigue causes motor units to discharge more synchronously (Mori 1973; Hunter and Enoka 2003; Holtermann et al. 2007). This could possibly influence the synchronization of TMS evoked discharges, and thus affect MEP size.

- Fourth, TMS drives some of the spinal motor neurons to discharge repetitively (Berardelli et al. 1991; Naka and Mills 2000; Z' Graggen et al. 2005). The amount of repetitive discharges is unpredictable ( $Z^{\prime}$ Graggen et al. 2005), may change during fatigue (Andersen et al. 2003), and may thus increase the MEP size in an unpredictable way.

In the present study we applied the TST (Magistris et al. 1998) to quantify central motor conduction during fatigue. The TST allows simultaneous assessment of the peripherally and centrally evoked responses. Moreover, it eliminates the effects of discharge desynchronization and repetitive discharges, thus allowing to estimate changes of the percentage of cortico-spinal motor neurons activated by the stimulus much more precisely than conventional MEPs (Magistris et al. 1998).

\section{Materials and methods}

\section{Subjects}

The experiments were performed in 23 healthy subjects, 5 women and 18 men, aged $21-54$ years (average 26 years); including some of the authors. None of the subjects had a history of previous neurological disorders, implanted metal in the eye or brain, or a cardiac pacemaker. The study was approved by the local ethics committee, and written informed consent was obtained from all subjects.

Six different experiments were done, and some of the subjects participated in two or more of them (summary of the experiments given in Table 1). In the following, the basic methods are described, and modifications of these basic methods are given for each of the six experiments thereafter.

\section{Electrophysiological and mechanical recordings}

Compound muscle action potentials were recorded from the left abductor digiti minimi muscle (ADM) using the muscle-belly tendon montage with surface electrodes (diameter $0.8 \mathrm{~cm}$ ). A ground electrode was taped to the dorsum of the hand. A Viking Select EMG apparatus was used for the recordings (Nicolet Biomedical, Madison, WI, USA). Bandpass filtering was $2 \mathrm{~Hz}-10 \mathrm{kHz}$. 
Table 1 Exercise protocols used in this study

\begin{tabular}{|c|c|c|c|c|c|c|}
\hline Experiment & $\begin{array}{l}\text { Number } \\
\text { of subjects }\end{array}$ & Type of exercise & Level of exercise & $\begin{array}{l}\text { Duration of } \\
\text { exercise (min) }\end{array}$ & Recovery period & Recording \\
\hline$\# 1$ & 13 & Voluntary & $100 \%$ of MVC & 2 & $9 \mathrm{~min}$ & TST test \\
\hline \#2 & 6 & Voluntary & $100 \%$ of MVC & 2 & $9 \min$ & TST control \\
\hline \#3 & 6 & Voluntary & $50 \%$ of MVC & 4 & $9 \mathrm{~min}$ & TST test \\
\hline \#4 & 7 & Imposed by el. stim. & $100 \%$ of motor units & 2 & $9 \min$ & TST test \\
\hline$\# 5$ & 6 & Voluntary & $100 \%$ of MVC & 2 & Cuff $2 \min +9 \min$ & TST test \\
\hline$\# 6$ & 10 & Voluntary & $100 \%$ of MVC & 2 & $9 \mathrm{~min}$ & Cortical silent period \\
\hline
\end{tabular}

Differences from the basic exercise Experiment \#1 are highlighted in bold

The isometric voluntary force of left little finger abduction was measured by placing the finger on a lever attached to a force transducer (Sensotec Inc., Columbus, Ohio USA). The subjects sat on a chair with their left forearm and hand fastened with Velcro straps to a platform in front of them, holding the force transducer. The force signal was DC-amplified using a Sedia amplifier (Sedia, Givisiez, Switzerland). It was sampled at $4 \mathrm{kHz}$ by a stand alone $\mathrm{AD}$ converter (MacLab, ADInstruments Pty Ltd., Castle Hill, NSW, Australia) connected to a personal computer (Macintosh, Apple Computer Inc., Cupertino, CA, USA) for later offline analysis. During the experiments, the force signal was fed into a digital oscilloscope in front of the subject, to allow visual feedback of the exerted force (Arányi et al. 1998). The system used did not allow measuring force in absolute units, but was calibrated to yield the force output in percent of maximal voluntary contraction (MVC).

Magnetic stimulation

Transcranial magnetic brain stimulation was performed using a Magstim 200 stimulator (Magstim Company Ltd., Spring-Gardens, Whitland, UK), with a circular 90-mm hand-held coil. The centre of the coil was at the vertex or slightly lateral toward the stimulated right hemisphere. Face 'B' was up. Slight coil displacements were made in all directions until the position yielding the lowest threshold was found. The coil was then kept in the same position throughout the experiment. The threshold was determined at rest as the stimulus intensity in percent of the maximal stimulator output that yielded a discernible response of the relaxed target muscle in 50\% of eight or ten trials at an amplification of $100 \mu \mathrm{V} /$ division (Rothwell et al. 1999).

Triple stimulation technique

The TST was described in detail previously (Magistris et al. 1998, 1999; Rösler et al. 2000). In short, it consists of a succession of three stimuli, namely (1) the transcranial magnetic brain stimulus, (2) a supramaximal stimulus of the ulnar nerve at the wrist, and (3) a supramaximal stimulus of the brachial plexus at Erb's point. These stimuli are appropriately timed to yield a TST test response. The events occurring as a result of the TST are depicted in Fig. 1a. In the context of the present study it is important to note that as a result of these stimuli, the trace includes an M-response to supramaximal ulnar nerve stimulation (i.e., the first deflection of the TST trace, Fig. 1a). In addition, it includes a second deflection, which relates in size to the number of spinal motor neurons that were driven to discharge by TMS (Fig. 1a). In previous studies, we quantified this second deflection by comparing the TST test response to a TST control response, obtained by three successive supramaximal stimuli given at (1) Erb's point, (2) the ulnar nerve at the wrist, and (3) Erb's point (Fig. 1b). The amplitude and area ratio of TST test:TST control gave the proportion of the spinal MNs supplying the target muscle which were brought to discharge by the TMS (Fig. 1b). Because the timing during the fatigue experiments did not allow recording of TST control responses, the quantification of the responses was modified, and the second deflection was directly compared to the first deflection (Fig. 1a). The resulting ratio was termed "central conduction index" (CCI). Compared to the TST test:TST control calculation, the CCI contains a small systematic error, which is caused by the small amount of desynchronisation during nerve conduction in the periphery, and by muscle-nerve ephaptic backfiring (Fig. 1; for a detailed discussion see Magistris et al. 1998). At the beginning and the end of each experiment, a TST control response was obtained to calibrate the CCI. Moreover, a separate series of control experiments were performed to exclude fatigue-induced changes of TST control responses (see below).

Experiment 1 (=basic experimental protocol; $n=13$ subjects)

Before the fatiguing exercise, MVC was determined as best of three trials. Then TST test responses and one TST control response were recorded, while the subjects performed a 


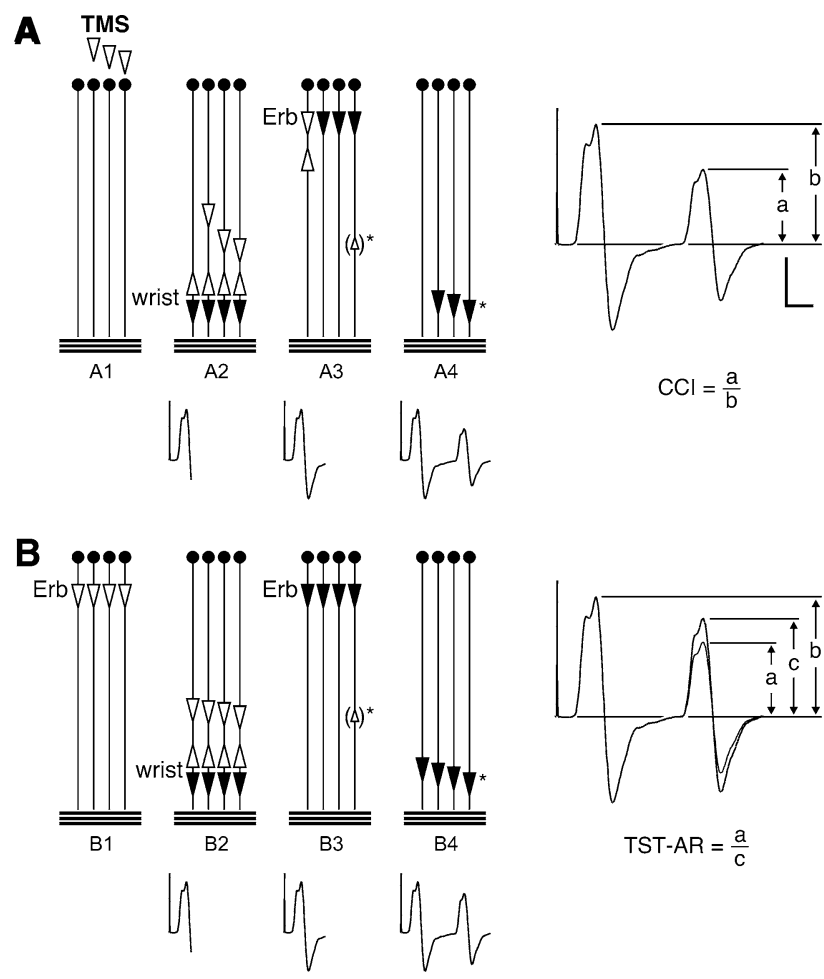

contraction of $20 \%$ of their maximal voluntary contraction force $(20 \%$ MVC). The minimal stimulus intensity was determined that evoked a TST amplitude ratio close to $100 \%$ (i.e., that nearly $100 \%$ of all MNs leading to the target muscle were excited). The stimulus intensity needed to reach such "maximal" responses were between 75 and $100 \%$ of the maximal stimulator output. The same stimulus intensity, rounded up to the next $5 \%$ was then used throughout the experiment. Background contractions of $20 \%$ MVC were chosen to facilitate the responses. In some experiments, especially during ischemia (see below), fatigue caused a drop in muscle force below $20 \% \mathrm{MCV}$. In these cases, TST stimulations were done during the maximal contraction the subject could produce (see, e.g., the experiment in Fig. 2). This was judged acceptable since facilitation saturates at levels well below 5\% MCV in unfatigued subjects, a force output always reached in our experiments. At these levels of contraction, small alterations of the contraction influence the size of the response only marginally (Rösler et al. 2002). The fatiguing exercise lasted $2 \mathrm{~min}$. Subjects performed repetitive maximal abductions of the little finger, of $1 \mathrm{~s}$ duration, every other second $(0.5 \mathrm{~Hz})$. A metronome gave the timing of the contractions. During these $2 \mathrm{~min}$, the subjects were asked every $15 \mathrm{~s}$ to perform a contraction at $20 \%$ of the initial MVC, and a TST test was performed (see upper panel in Fig. 2). Following the exercise, the TST test was repeatedly performed during short contractions of $20 \%$ of the initial MVC, after 15, 30, 45, 60, 120, 240, and $420 \mathrm{~s}$, to assess changes during recovery. The maximal voluntary force was determined at each of these time points.
Fig. 1 Triple stimulation technique (TST) principle. The motor tract is simplified to four spinal motor neurons (MNs); horizontal lines represent the abductor digiti minimi (ADM) muscle fibres supplied by the four neurons. Black arrows depict action potentials (APs) that cause a trace deflection, open arrows those that do not. Below, the trace recording is given at each time point. a TST test recording and calculation of the central conduction index (CCI); $A 1$ submaximal TMS excites three of four MNs. A2 On 3/4 MNs, TMS induced APs descend. Desynchronization of the three APs has occurred. After a delay, a maximal stimulus is applied to the ulnar nerve at the wrist. It gives rise to a first negative deflection of the recording trace. The antidromic APs collide with the descending APs on MNs 2, 3, and 4. The AP on MN 1 continues to ascend. A3 After a second delay a maximal stimulus is applied to the brachial plexus at Erb's point. On MN 1, the descending AP collides with the ascending AP. On neurons $2-4$, no collision occurs, and APs descend. During their descent, a minor degree of desynchronization occurs, as typical for peripheral nerves. In some subjects, ephaptic muscle-nerve backfiring will occur (small open arrow in brackets with asterisk), causing an AP to ascend on neuron 4. A4 APs on MNs 2-4 evoke a muscle response. Note that MNs 2-4 were those initially excited by the transcranial stimulus. The response is recorded as the second deflection of the TST test trace. It will be a bit smaller than the first deflection because some desynchronisation of APs will occur during conduction from Erb's point to the wrist, and because backfiring ascending APs (small open arrow in brackets with asterisk) may collide with some of the descending APs. The CCI is calculated as the ratio of second and first deflection $(\mathrm{CCI}=\mathrm{a} / \mathrm{b}$; right panel, calibration $5 \mathrm{mV} / 5 \mathrm{~ms}$ ). b TST control recording and calculation of the TST amplitude ratio (TST-AR); $B 1$ a maximal stimulus is applied at Erb's point. $B 2$ After a delay, a maximal stimulus at the wrist causes the first deflection of the TST control trace. B3 After a delay a maximal stimulus is applied at Erb's point, evoking action potentials on all neurons. During their descent, a minor degree of peripheral desynchronization occurs, matching (and calibrating) the desynchronisation that occurred during the TST test procedure. If muscle-nerve backfiring occurs (small open arrow in brackets with asterisk), it will also match that during the TST test procedure. B4 A response from the four MNs is recorded as the second deflection of the TST control trace. It will be smaller than the first deflection, caused by peripheral desynchronisation and ephaptic backfiring. The test response is quantified as the ratio of TST test:TST control responses, thereby eliminating influences by peripheral desynchronisation and backfiring $(\mathrm{TST}-\mathrm{AR}=\mathrm{a} / \mathrm{c}$; right panel, same calibration as in a)

Experiment 2 ( $n=6$ subjects)

Served to rule out contributions of peripheral conduction changes to the second (i.e., the "central") trace deflection of the TST. The fatiguing exercise protocol was the same as in Experiment 1 . However, the TST test stimuli were replaced by TST control stimuli throughout (brain stimulation replaced by proximal nerve stimulation at Erb's point; Fig. 1b).

Experiment 3 ( $n=6$ subjects)

The influence of the level of exertion was assessed. Subjects performed contractions of 50\% MVC (instead of $100 \%$ ), and the exercise lasted 4 min (instead of 2). During exercise, a TST test recording was done every $15 \mathrm{~s}$ during a contraction of $20 \%$ MVC. The post-exercise protocol was the same as in Experiment 1. 
Fig. 2 Mechanical force recording of little finger abduction of one subject during Experiment 5 (exercise-cuffrecovery). Note that the time axes is different in the two panels. Small arrows indicate TST procedures
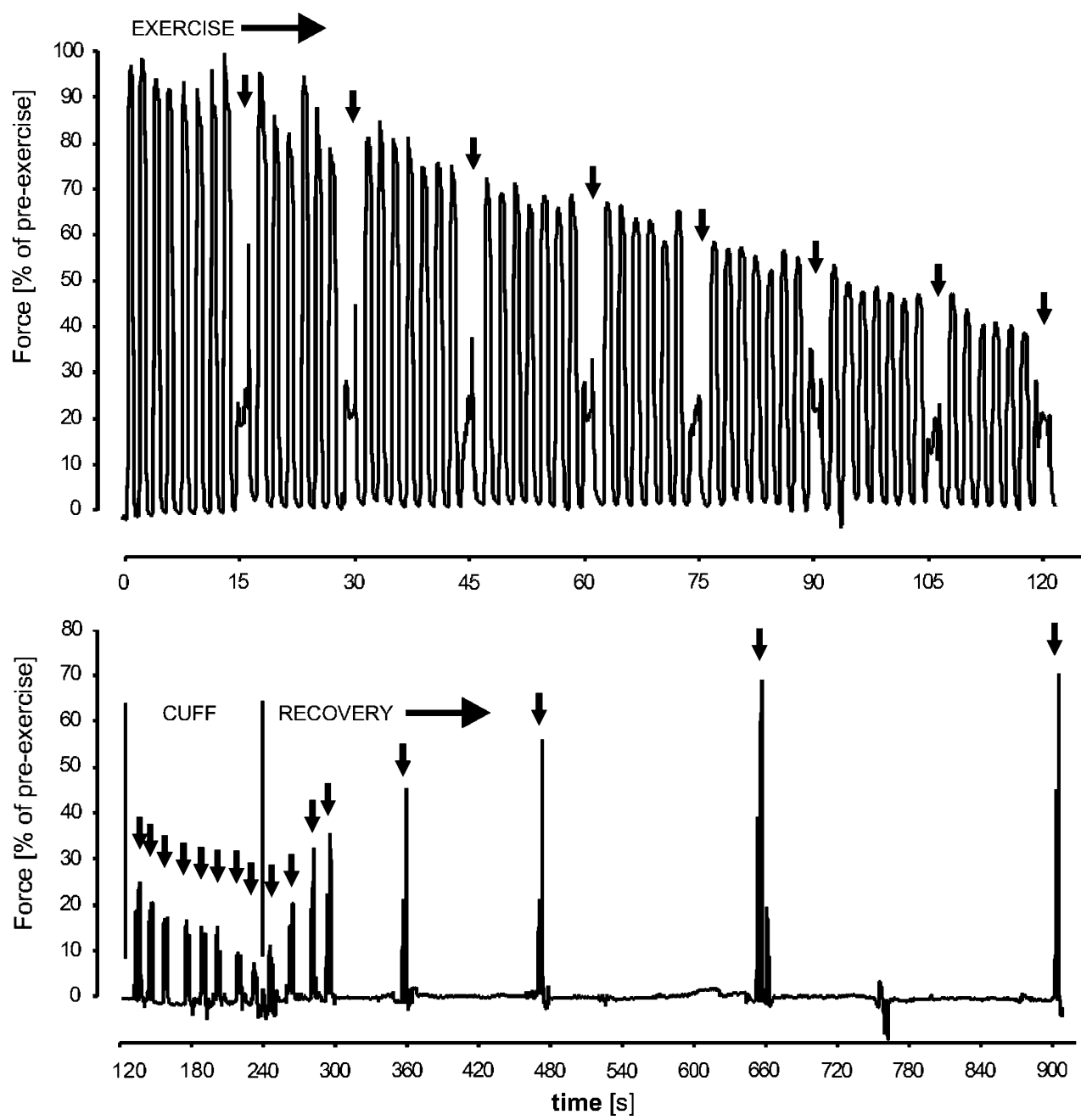

Experiment 4 and 5 served to assess the influence of peripheral feedback and voluntary activation on the CCI.

Experiment 4 ( $n=7$ subjects)

The maximal voluntary contractions of the subjects were replaced by tetanic supramaximal stimuli of the ulnar nerve at the wrist (Stokes et al. 1989; Garland and McComas 1990). Stimulus trains of $20 \mathrm{~Hz}$ of $1 \mathrm{~s}$ duration each were given every other second, for a total time of $2 \mathrm{~min}$. These fatiguing stimuli were interrupted every $15 \mathrm{~s}$, to perform a TST test procedure, during which the subjects performed a voluntary contraction of $20 \%$ of their initial MVC. The recovery was assessed as in Experiments 1 and 3.

\section{Experiment 5 ( $n=6$ subjects)}

Two minutes of voluntary exercise at $100 \%$ MVC were followed by $2 \mathrm{~min}$ in which the circulation was blocked by use of a cuff, as described by Bigland-Ritchie et al. (1986). During the 2 min of hemostasis, subjects remained principally relaxed, but maximal force measurements and TST test procedures at $20 \% \mathrm{MVC}$ were performed every $15 \mathrm{~s}$. After opening of the cuff, recovery was assessed as in Experiments 1 and 3 .

Experiment 6 ( $n=10$ subjects $)$

Done to assess intracerebral inhibitory mechanisms. The fatiguing exercise and the timing of the stimuli and force measurements was performed as in Experiment 1. Instead of the TST, single TMS were given, during background contractions of $20 \%$ of the initial MVC. The stimulus intensity was chosen to evoke "maximal" MEPs (i.e., the MEPs appeared not to increase when the stimulation intensity was further increased). The cortical silent period (CSP, i.e. the period of interruption of the background EMG) was measured. In addition, this experiment allowed measurements of the amplitude of the MEP preceding the CSP.

\section{Statistical analysis}

All measurement results were expressed in percentage of pre-exercise levels (pre-exercise maximal force and 
pre-exercise $\mathrm{CCI}=100 \%$ ). To test differences of mean, nonparametric tests (Wilcoxon or Mann-Whitney $U$ ) were applied throughout, as many of the measured parameters were not distributed in a Gaussian way.

\section{Results}

By using high TMS intensity and a constant facilitatory background muscle contraction of $20 \%$ of the initial MVC, we intended to achieve excitation of all or nearly all MNs leading to the ADM in our subjects. Before the start of the exercise, the proportion of motor units responding to TMS was high in all subjects. The TST amplitude ratio [i.e., the ratio of the TST test to the TST control amplitude (Magistris et al. 1998)] is indicative of the proportion of motor units discharging in response to TMS (Fig. 1). Averaged across all experiments it was $96 \%$ before exercise, and $93 \%$ after recovery. The average CCI was 0.92 before exercise, and 0.90 after recovery at the end of the experiment. The difference between CCI and TST amplitude ratio was $-4.7 \%$ before exercise, and $-3.3 \%$ after exercise (not statistically significant). The difference between TST amplitude ratio and CCI resides in the periphery. We conducted Experiment 2 to test if peripheral changes affected the results during our exercise protocol, by performing TST control measurements throughout the fatigue protocol. No evidence of peripheral changes influencing the CCI measurements during fatigue were found. Hence, the CCI reflects the amount of central conduction during fatigue. To account for the small systematic error, which differed somewhat between subjects, we normalized the measured CCIs to the individual pre-exercise $\mathrm{CCI}=100 \%$.

\section{Experiment 1}

During exercise, muscle force decreased virtually linearly in all subjects, reaching approximately $40 \%$ of MVC after 2 min of exercise (Fig. 3a). Recovery of muscle force was slow, and most subjects did not recover fully during the post-exercise interval of $11 \mathrm{~min}$ (Fig. 3a). The CCI decreased markedly in all subjects during exercise. The initial CCI-decline during the first minute of exercise was significantly steeper than that of the muscle force $(P<0.05$; Fig. 3a). During the second minute of exercise, the CCI plateaued at a level of approximately $40 \%$ of the pre-exercise value. After the exercise, the CCI recovered faster than force, and averaged pre-exercise levels 4 min after the exercise (Fig. 3a). An example of TST recordings in one subject is given in Fig. 4. It is noteworthy that the degree of CCI reduction differed markedly between subjects, whereas the reduction of force was less variable. Therefore, the relation between force reduction and CCI reduction varied consid- erably between the subjects, as demonstrated by two examples in Fig. 5. Moreover, CCIs varied sometimes from stimulus to stimulus (e.g., in Figs. 4, 5), while the individual force reduction was considerably less variable. In pilot experiments in several subjects, we attempted to overcome the exercise related CCI reduction by increasing the TMS intensity by $10-20 \%$. The stronger stimuli never appeared to change the CCI beyond the variability seen during experiments with constant stimulus output.

\section{Experiment 2}

This experiment served to assess the possibility of influences of peripheral conduction changes on the CCI. The TST test procedures were replaced with TST control procedures (stimuli: Erb-wrist-Erb). Amplitudes and areas were quantified in analogy to the CCI by calculating the ratio of the second to first deflection size (Fig. $1 \mathrm{~b}$ : c/b). This ratio remained constant in all subjects, thus ruling out an influence of peripheral conduction changes on the $\mathrm{CCI}$ during the fatigue protocol.

\section{Experiment 3}

In this experiment, subjects performed repetitive contractions of 50\% MVC, to assess the influence of the exertion level on force and CCI. To reach a comparative level of motor fatigue as in Experiment 1, the exercise duration was increased to 4 min (Fig. 3b). Subjectively, subjects participating in both experiments usually considered the fatigue experienced in Experiment 3 greater than that in Experiment 1 . The CCI reduction rate was significantly less step than in Experiment 1 (Fig. 3), although greater subjective fatigue, similar muscular force loss, and similar maximal force output at the end of the exercise were observed. After $120 \mathrm{~s}$ of exercise with $50 \%$, the mean CCI was $79 \%$ (SD $26 \%$ ), while in Experiment 1 (exercise of 100\%), it was $46 \%$ (SD 13\%; $P<0.025$ ). Only after 4 min of exercise at $50 \%$, the CCI was $51 \%$, which was not statistically different from the CCI at the end of the second minute of exercise in Experiment 1 . Recovery of force and of CCI after exercise was similar as in Experiment 1.

\section{Experiment 4}

To test if voluntary drive was required for the reduction of $\mathrm{CCI}$, or if it was caused by a reflex mechanism from the periphery, we replaced voluntary contractions by tetanic stimulation of the target muscle ("stimulated exercise"). The subjects judged this procedure unpleasant but sustainable. Our force measuring device did not allow to record force output in absolute units, so that the force produced by the stimulated muscle contractions could not directly be 
Fig. 3 CCI (black squares) and maximally achieved muscle force (white circles) during fatigue and recovery. Given are mean and standard error of mean. a Experiment 1: exercise at $100 \%$ MVC for $2 \mathrm{~min}$. Asterisks indicate significant difference between normalized force and normalized CCI. b Experiment 3: exercise at 50\% MVC for $4 \mathrm{~min}$; c Experiment 4 : imposed exercise by tetanic electrical stimulation, $20 \mathrm{~Hz}$ trains of $1 \mathrm{~s}$ duration at $0.5 \mathrm{~Hz}$, for $2 \mathrm{~min}$; arrows indicate significant difference of CCI from pre-exercise levels. d Experiment 5: exercise at $100 \% \mathrm{MVC}$ for $2 \mathrm{~min}$; cuff inducing venous stasis for $2 \mathrm{~min}$, recovery. Note that all protocols cause a reduction of muscle force to approximately $40 \%$ after 2 or 4 min
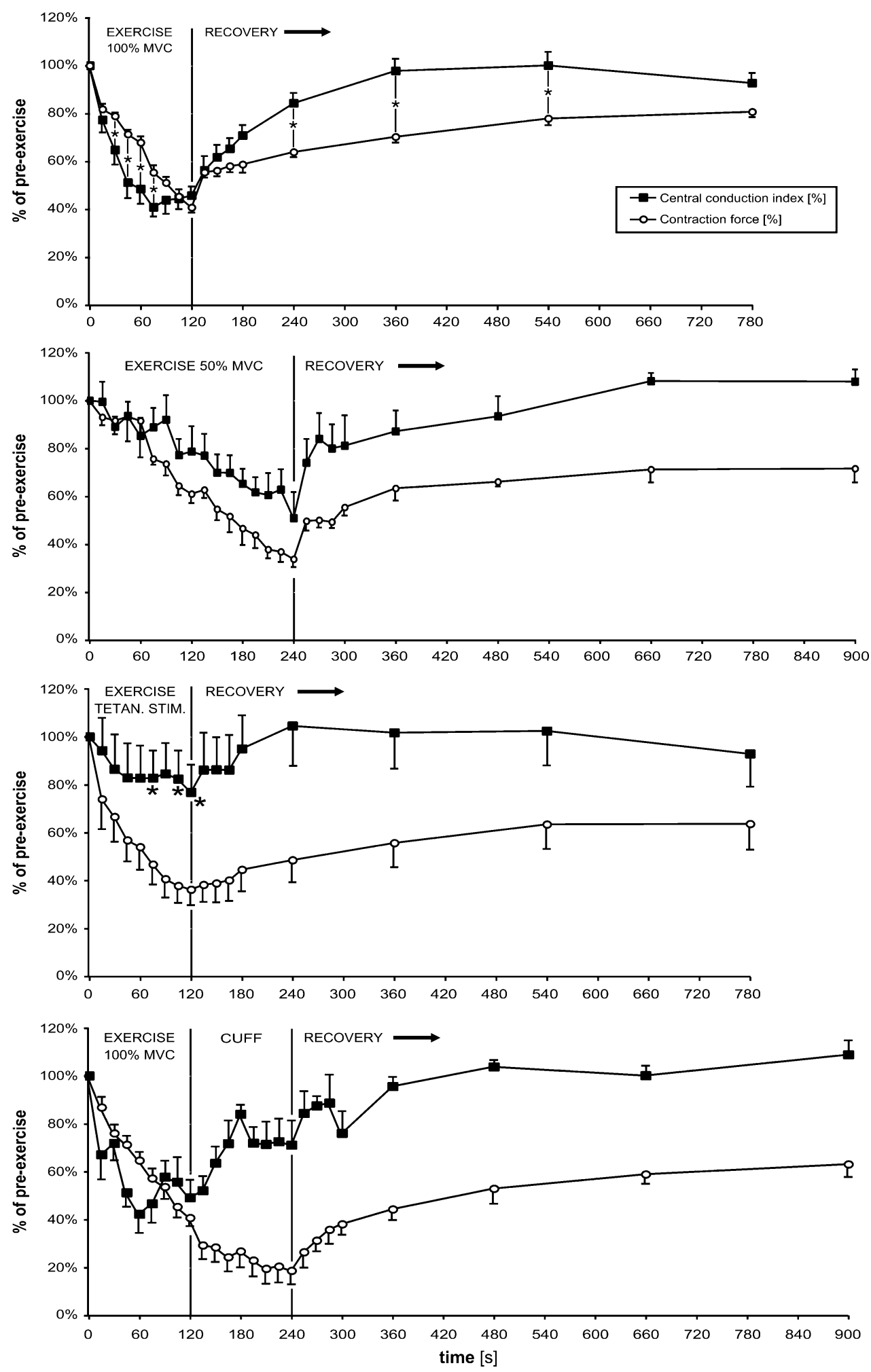

compared with to that of voluntary contractions. The decrease of force was however very similar to that observed with voluntary contractions (Fig. 3c). At the same time the CCI decreased significantly less than during voluntary exercise (Fig. 3c). During the second minute of exercise, however, it was significantly reduced as compared to the pre-exercise level. Recovery in Experiment 4 was a bit slower than in Experiments 1 and 3, possibly because of the more profound fatigue induced by the stimulation procedure (Fig. 3). 
Fig. 4 TST recordings of one subject during Experiment 1. Note the broadening of the M-wave (first deflections) during exercise, caused by peripheral fatigue. The second deflection quantifies central conduction. It decreases during fatigue and increases to preexercise levels during recovery. Calibration $5 \mathrm{mV} / 5 \mathrm{~ms}$

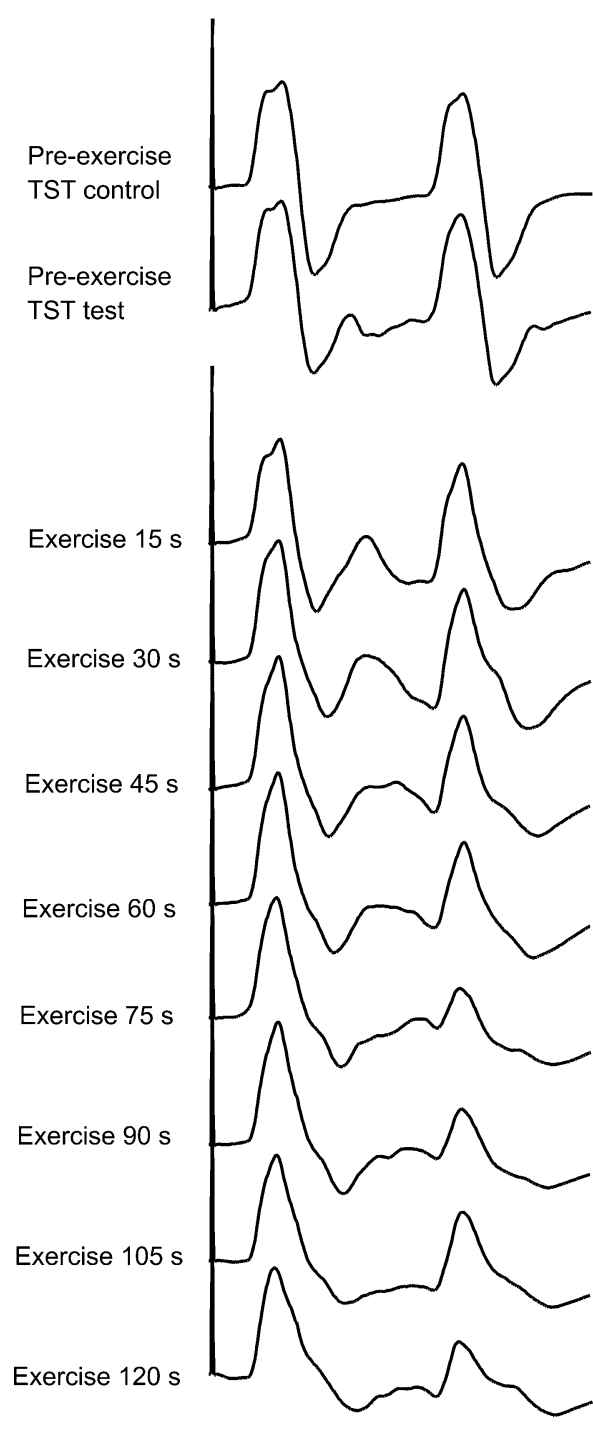

\section{Experiment 5}

After 2 min of exercise, a sphygmomanometer cuff was inflated around the upper arm, as described previously (Bigland-Ritchie et al. 1986), to induce ischemia. With this procedure, fatigue induced metabolites are kept within the muscle and muscle force does not recover. Force decline and CCI reduction during $2 \mathrm{~min}$ of exercise were similar to those in Experiment 1; with some more variability of the CCI (Fig. 3d). In the 2 min of muscle hemostasis, during which the only exercise consisted of one maximal contraction every 15 s followed by a $20 \%$ contraction to record the TST, force did not recover but continued to decrease (Fig. 3d). The CCI, however recovered quickly to similar levels as during the first 2 min of recovery in Experiment 1 (Fig. 3). Thus a similar dissociation between force and CCI occurred as in Experiment 4.

\section{Experiment 6}

The CSP is thought to be associated with intracortical inhibitory mechanisms which could contribute to central fatigue. In our ten subjects, the CSP increased significantly during the exercise (Fig. 6), and recovered quickly thereafter. The average pre-exercise silent period was $195 \mathrm{~ms}$ (SD $42.8 \mathrm{~ms}$ ), it increased to a maximum of $261 \mathrm{~ms}$ (SD $72.2 \mathrm{~ms}$ ) at the end of the exercise, and decreased to $216 \mathrm{~ms}$ (SD $56.0 \mathrm{~ms}$ ) after recovery. While the earlier part of the silent period is attributed to both, spinal and cortical mechanisms, the latter part after some $100 \mathrm{~ms}$ has been shown to result from intracortical inhibition (Fuhr et al. 1991). The average MEP amplitude before exercise was $5.1 \mathrm{mV}$ (SD $1.74 \mathrm{mV}$ ). It increased to a maximum of $6.1 \mathrm{mV}$ (SD $1.95 \mathrm{mV}$ ) after $75 \mathrm{~s}$ of exercise, and decreased to $4.2 \mathrm{mV}$ $(\mathrm{SD} 1.80 \mathrm{mV}$ ) at the end of the $11 \mathrm{~min}$ recovery period. The 


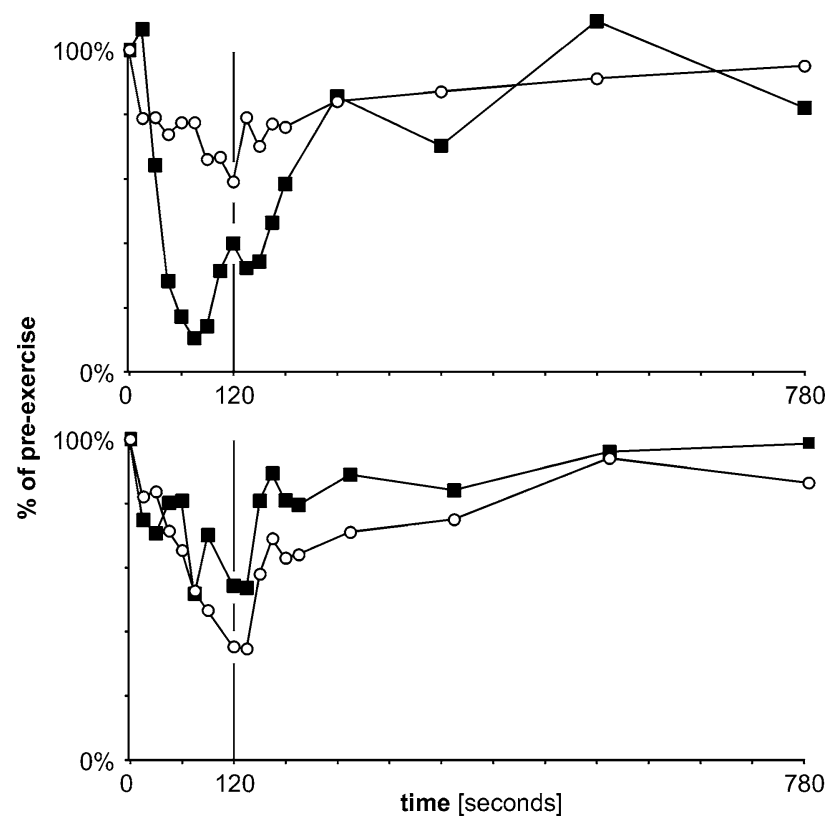

Fig. 5 CCI (black squares) and force (white circles) in two different subjects during Experiment 1. Note the marked interindividual difference in the relation between force and CCI

increase of MEP amplitude varied between subjects and reached statistical significance only at $15 \mathrm{~s}$ (Wilcoxon $P<0.05$ ) (Fig. 6).

\section{Discussion}

By use of the TST, it was previously shown that TMS fails to activate the entire spinal motor neuron pool during muscular fatigue (Andersen et al. 2003). This activation failure was related to the duration and level of exercise, suggesting that it reflected alterations of the corticomotor excitability and, possibly, the loss of force producing ability (Andersen et al. 2003).

In the present study, we characterized this phenomenon further. Using similar methods, our study confirms the exercise related drop in TST response size, as described by Andersen et al. (2003). As an extension to that study, our present data indicate that the relation between the reduction of CCI and the observed muscular fatigue is complex. While the reduction of force was rather uniform in all subjects, the exercise-related CCI depression varied markedly. These marked inter-individual differences would have been obscured by averaging across subjects, as in the study of Andersen et al. (2003). Moreover, the time course of the force decline and that of the CCI depression differed significantly, and the relation between decreases of CCI and force were not the same at different levels of exercise. Finally, our study suggests that CCI reduction is particularly large when voluntary drive (i.e., voluntary cortico-spinal activity) is involved during an exercise.

Exercise protocol and triple stimulation technique during fatigue

A number of exercise protocols were previously used in studies of central fatigue. We used a repetitive contraction protocol to circumvent hemostasis induced by intramuscular increases of tissue pressure related to sustained contraction. Pilot experiments and the study by Andersen et al. (2003) demonstrated that sustained work principally lead to
Fig. 6 Cortical silent period (black squares), MEP amplitudes (white squares) and muscle force (white circles) during 2 min of exercise at $100 \%$ MVC and recovery (Experiment 6). Asterisks denote significant difference from pre-exercise values

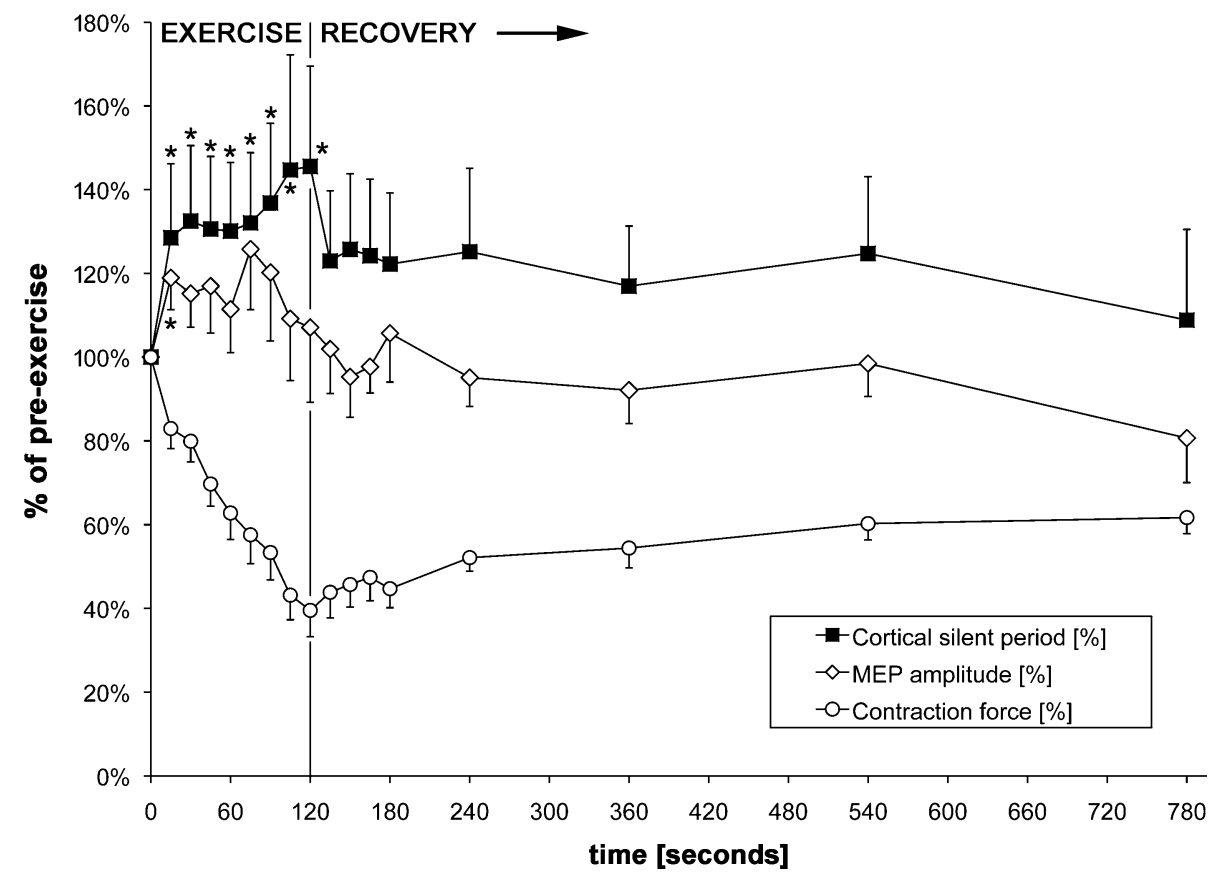


similar alterations in central motor conduction as those observed here. To achieve activation of $100 \%$ of MNs by TMS, facilitation by voluntary contraction is usually necessary (Magistris et al. 1998; Rösler et al. 2002), and the relation between the amount of pre-contraction and increase of the TMS response is not linear in ADM (Hess et al. 1987; Rösler et al. 2002). To avoid the need for calibration of the facilitatory pre-contraction, some previous fatigue studies performed TMS with the relaxed muscle. The disadvantage of this approach is that responses are usually not maximal (i.e., not all spinal MNs are involved in the examination). Other studies used maximal force (MVC) to facilitate the responses. The main disadvantage of this approach is that due to the high level of background activity, the measurement of responses becomes less precise (Uozumi et al. 1991). In addition, even if MVC is used to facilitate responses, conventional MEPs do not allow controlling that all target MNs are driven to discharge. In the present study we chose contractions of $20 \%$ of MVC to facilitate the TST responses throughout the exercise protocol. With this amount of pre-contraction, activation of nearly $100 \%$ of spinal motor neurons is usually achieved in non-fatigued healthy subjects, using the MagStim 200 stimulator (Rösler et al. 2002). Small changes of the level of pre-contraction will not critically affect the TMS responses, because the facilitatory effect on MEPs (Hess et al. 1987) and TST responses (Rösler et al. 2002) nearly saturates at contraction levels of 5\% and more (i.e., increasing the pre-contraction force does not lead to a significant further increase of the response size). During muscular fatigue, the sequence of motor units that cease contracting is fairly fixed; and in particular, units that were recruited last with increasing levels of work will fatigue first (Zajac and Faden 1985; Carpentier et al. 2001). Hence, it is reasonable to assume that in most of our experiments, during fatigue, the same pool of MNs was activated for the facilitatory maneuver; and that spinal facilitation was thus sufficient. In the experiment using muscle ischemia (Experiment 5), muscle force dropped below $20 \%$ of the initial MVC in some subjects. At this point, the facilitatory contraction had to be maximal. Since maximal voluntary contraction may be associated with more complex excitability changes along the motor pathway (e.g., by increased voluntary drive to overcome the limitation, associated with increased cortical excitability and greater MN firing rates), it becomes difficult to estimate the effect of facilitation in this phase of Experiment 5 . However, the CCI recovered in all six subjects despite ischemia, indicating that the $\mathrm{CCI}$ recovered during muscle ischemia, even though the conditions of facilitation had to be altered.

Most of the technical implications of the TST have been discussed previously (Magistris et al. 1998; Rösler et al. 2002; Andersen et al. 2003). The TST allows a quantifica- tion of the number of spinal motor neurons driven to discharge by the transcranial stimulus. We demonstrated previously that the TST allows detection and quantification of even small changes of central conduction, which remain undetected by conventional MEPs (Rösler et al. 1999; Humm et al. 2004). This quantification becomes possible by eliminating influences of desynchronisation of the TMS induced motor neuron discharges and that of repetitive discharges on the response size. The voluntary activity necessary to facilitate the TST response may alter the TST deflections only marginally. The measuring error with a pre-contraction of $20 \% \mathrm{MVC}$ is maximally some $3-6 \%$, assuming physiological discharge rates and refractory times and considering the time slots in which voluntarily induced action potentials can enter the collisions involved. Hence, the measuring error induced by voluntary activity was probably negligible in the present experiments. In all previous TST studies, the exact quantification of the TST response was achieved by comparing a TST test response (obtained by TMS followed by peripheral stimulations of the muscle efferent at wrist and brachial plexus; Fig. 1a), with that of a TST control response (obtained by proximal peripheral brachial plexus stimulation, followed by stimulation at wrist and brachial plexus; Fig. 1b) (Magistris et al. 1998; Andersen et al. 2003). The multiple stimulation procedures for such a comparison appeared too time consuming during a fatigue protocol. Therefore we simplified the quantification by comparing the first and second deflection of the TST test response, yielding the CCI (Fig. 1a). With this simplification, a systematical error is introduced into the measure, since the size of the second TST deflection is usually somewhat smaller than the first one, due to peripheral mechanisms, in particular by muscle-nerve ephaptic backfiring and peripheral desynchronisation (Magistris et al. 1998). In our experiments, this difference originating in the periphery was small, averaging some $3-4 \%$, and did not change with fatigue (see below). We normalized the $\mathrm{CCI}$ during fatigue to the pre-exercise values, in order to compensate for this small systematic error. An additional advantage of this normalization was that a direct comparison of the time course of force and CCI during exercise and recovery could be done, since force was also expressed in percent of the pre-exercise value.

Remarkably, while the CCI decreased during exercise, the size of the conventional MEPs did not (there was even a tendency to increase during exercise, which reached statistical significance at $t=15 \mathrm{~s}$, Fig. 6). Increases of the MEP during fatiguing muscular exercises have been observed previously (Taylor et al. 1996, 1999), and a dissociation between MEP- and TST-response size was also reported (Andersen et al. 2003). MEP increases were attributed to an increase in excitability of neurons in the motor cortex (see, e.g., Taylor and Gandevia 2008). The dissociation between 
MEP- and CCI size indicates that during fatigue, the synchronization of TMS-induced motor neuron discharges changed, or the number of repetitive motor neuron discharges changed, or both. A single cortical stimulus can induce multiple discharges of the spinal motor neuron, which may increase the amplitude of the MEP, but not that of the TST response, because they do not participate in the collisions during the TST sequence (for a discussion see Z'Graggen et al. 2005). In our experience, the effect of repetitive motor neuron discharges on MEP size is rather small, at least in the non-fatigued muscle (Z'Graggen et al. 2005). Repetitive discharges can be roughly estimated from the TST recordings, where they occur between the two main deflections (Magistris et al. 1998; Bühler et al. 2001) but re-inspection of the present traces was inconclusive in this respect. We are currently conducting experiments to address this question. On the other hand, spinal motor neuron discharges after TMS may be desynchronized, depending on transmissional events at the spinal level, and alsoyet probably to a lesser degree-on the intracortical transmission onto the cortico-spinal path. The effect of discharge desynchronization on MEP size is important, reducing amplitude and area by one to two-thirds, in an unpredictable and variable manner (Magistris et al. 1998; Rösler et al. 2002). On the same token, improving synchronization of the discharges could potentially increase MEP size quite considerably. Indeed, muscle fatigue is associated with an increased synchronization of motor unit discharges, as shown in coherence studies (Mori 1973; Hunter and Enoka 2003; Holtermann et al. 2007). It is believed to be linked to the occurrence of fatigue tremor. We have observed dramatic increases in TST amplitudes during cold induced tremor, supporting the possibility of such a mechanism (Rösler and Humm, unpublished results).

\section{Peripheral fatigue}

During our exercise protocol, we always observed changes of the response configuration typical for peripheral fatigue, e.g., a broadening of the muscle CMAP, sometimes accompanied by a small amplitude reduction (Fig. 4). Our Experiment 2 confirmed that this peripheral effect was present in both deflections of the TST curve in parallel, when the brain stimulus was replaced by a proximal peripheral nerve stimulus (Experiment 2). Thus the ratio between second and first deflection remained constant throughout the entire exercise protocol in Experiment 2. The observed changes of CCI with TMS (Experiments 1, 3, 4) were therefore caused by changes of central conduction, and were not influenced by peripheral effects of fatigue.

In Experiment 4, we replaced voluntary muscle contractions by trains of tetanic stimulation of the ulnar nerve at the wrist. These stimulus trains were given to resemble as much as possible the voluntary activation; e.g., using a relatively "physiological" frequency of $20 \mathrm{~Hz}$ (Bigland-Ritchie et al. 1986; Pitcher and Miles 2002) in the same duration and rhythm than the bouts of voluntary contraction. As a result, the decline in force was very similar to that seen with voluntary contractions (Fig. 3), demonstrating that peripheral fatigue was sufficient to cause the observed loss of force in either experiment. It is then reasonable to assume that the limiting factor for force production during and after fatigue, caused in this manner, was located in the periphery.

\section{Changes of central conduction during fatigue}

Our data confirm that during fatigue the loss of muscle force is roughly paralleled by changes of TMS-evoked central motor output (as measured by the CCI). Hence, during the reduction of muscle force, fewer motor neurons responded to TMS, suggesting that they became less responsive to synaptic input (Taylor and Gandevia 2008). Our quantitative analysis revealed a dissociation of force and CCI during fatiguing muscle activity. This dissociation was demonstrated in the time course during exercise and recovery (Fig. 3a), between subjects (Fig. 5), and particularly for exercise with different workloads (Fig. 3a vs. b). It suggests that the decreasing number of motor units accessible to TMS does not fully explain the loss of force during motor fatigue.

The observed reduction of CCI could be caused by mechanisms located at different sites. Our experiments suggest an important supraspinal contribution to this phenomenon. First, cortical inhibitory phenomena are suggested by the lengthening of the CSP, which was observed here (Fig. 6) and by others (Andersen et al. 2003; Benwell et al. 2006, 2007; Taylor et al. 1999). It is remarkable that the time course of the CSP prolongation was similar to that of the exercise induced CCI reduction (Figs. 3a, 6). Hence, cortical inhibitory phenomena occur during fatigue and may contribute to reduce the excitability of corticospinal cells, reducing the response to TMS. Second, our Experiment 4 demonstrates that the CCI drops only little if voluntary drive is not involved. When the muscles were driven to fatigue by peripheral nerve stimulation, the muscular force fatigued similarly as during the voluntary exercises, yet the CCI decreased only little (Fig. 3). Hence voluntary drive appears important for the drop of CCI to occur during fatigue. Repetitive activation may cause refractoriness of the peripheral motor axons (Vagg et al. 1998), but there is little to suggest that the electrically induced action potentials led to substantially different levels of refractoriness than the maximal voluntary activation. Hence voluntary drive appears important for the drop of CCI to occur during fatigue, suggesting that the source of most of the reduction of the CCI is located supraspinally. Both, inhibitory intracortical phenomena or descending inhibitory input on 
alpha-motor neurons during fatigue are conceivable, but our data do not allow to discriminate between these possibilities. During a sustained maximal voluntary contraction, an additional superimposed twitch can be evoked by motor cortex stimulation. The size of this twitch increases during fatigue and suggests an increasing failure at the supraspinal level to generate all of the possible motor cortical output (Taylor and Gandevia 2008). It is possible that our present observation corresponds to this phenomenon.

A reduction of spinal motor neuron excitability was previously shown during fatiguing exercises, and could possibly also affect the size of the CCI. A reduction in motor neuron discharge rates during fatigue is caused by reflex inhibition of alpha-motor neurons by group III and IV muscle afferents, as evidenced by sustained post-exercise depression of discharge rates during hemostasis (Bigland-Ritchie et al. 1986; Woods et al. 1987). In the present study, the recovery of CCI was not delayed by hemostasis (Experiment 5, Fig. 3d); and an undisturbed recovery also of conventional MEP amplitudes after sustained post-exercise muscle ischemia was reported previously (Taylor et al. 2000). In addition, the observed time course of the CCI reduction, which occurred right from the beginning of the exercise and eventually plateaued during the second minute of exercise (Fig. 3a) would not be compatible with accumulation of metabolites during exercise, which occurs exponentially (Hampson et al. 2001). Taken together, most of the observed CCI decrease was not mediated via ischaemically sensitive group III and IV muscle afferents. On the other hand, we observed a small but significant reduction of CCI when the muscle was driven to fatigue by tetanic stimulation via the peripheral nerve (Experiment 4, Fig. 3c), compatible with some afferent influences on the CCI. Recurrent inhibition and reduced afferent input from Golgi apparatus and muscle spindles are possible sources of this inhibition (Kukulka et al. 1986; Zytnicki et al. 1990; Pettorossi et al. 1999). Our stimulation study is at contrast to previous studies, which observed facilitation of MEPs or alpha-motoneurones after peripheral nerve stimulation (Date et al. 1991; Ridding et al. 2000; Pitcher and Miles 2002). These differences are possibly related to different stimulation protocols, different target muscles, and the use of the TST eliminating repetitive charges from the compound response. Summarized, our data suggests an important supraspinal contribution to the drop of the CCI during fatigue, while the contribution of spinal mechanism appears rather small.

\section{References}

Andersen B, Westlund B, Krarup C (2003) Failure of activation of spinal motoneurones after muscle fatigue in healthy subjects studied by transcranial magnetic stimulation. J Physiol 551:345-356
Arányi Z, Mathis J, Hess CW, Rösler KM (1998) Task-dependent facilitation of motor evoked potentials during dynamic and steady muscle contractions. Muscle Nerve 21:1309-1316

Benwell NM, Sacco P, Hammond GR, Byrnes ML, Mastaglia FL, Thickbroom GW (2006) Short-interval cortical inhibition and corticomotor excitability with fatiguing hand exercise: a central adaptation to fatigue? Exp Brain Res 170:191-198

Benwell NM, Mastaglia FL, Thickbroom GW (2007) Differential changes in long-interval intracortical inhibition and silent period duration during fatiguing hand exercise. Exp Brain Res 179:255-262

Berardelli A, Inghilleri M, Rothwell JC, Cruccu G, Manfredi M (1991) Multiple firing of motoneurones is produced by cortical stimulation but not by direct activation of descending motor tracts. Electroencephalogr Clin Neurophysiol 81:240-242

Bigland-Ritchie BR, Dawson NJ, Johansson RS, Lippold OC (1986) Reflex origin for the slowing of motoneurone firing rates in fatigue of human voluntary contractions. J Physiol 379:451-459

Brasil-Neto JP, Pascual-Leone A, Valls-Sole J, Cammarota A, Cohen LG, Hallett M (1993) Postexercise depression of motor evoked potentials: a measure of central nervous system fatigue. Exp Brain Res 93:181-184

Bühler R, Magistris MR, Truffert A, Hess CW, Rösler KM (2001) The triple stimulation technique to study central motor conduction to the lower limbs. Clin Neurophysiol 112:938-949

Carpentier A, Duchateau J, Hainaut K (2001) Motor unit behaviour and contractile changes during fatigue in the human first dorsal interosseus. J Physiol 534:903-912

Date M, Schmid UD, Hess CW, Schmid J (1991) Influence of peripheral nerve stimulation on the responses in small hand muscles to transcranial magnetic cortex stimulation. Electroencephalogr Clin Neurophysiol Suppl 43:212-223

Edwards RH, Hill DK, Jones DA, Merton PA (1977) Fatigue of long duration in human skeletal muscle after exercise. J Physiol 272:769-778

Fuhr P, Agostino R, Hallett M (1991) Spinal motor neuron excitability during the silent period after cortical stimulation. Electroencephalogr Clin Neurophysiol 81:257-262

Gandevia SC, Petersen N, Butler JE, Taylor JL (1999) Impaired response of human motoneurones to corticospinal stimulation after voluntary exercise. J Physiol 521((Pt 3)):749-759

Garland SJ, McComas AJ (1990) Reflex inhibition of human soleus muscle during fatigue. J Physiol 429:17-27

Hampson DB, St Clair Gibson A, Lambert MI, Noakes TD (2001) The influence of sensory cues on the perception of exertion during exercise and central regulation of exercise performance. Sports Med 31:935-952

Hess CW, Mills KR, Murray NM (1987) Responses in small hand muscles from magnetic stimulation of the human brain. J Physiol 388:397-419

Holtermann A, Grönlnd C, Karlsson JS, Roeleveld K (2007) Motor unit synchronization during fatigue: described with a novel sEMG method based on large motor unit samples. J Electromyogr Kinesiol 19:232-241

Humm AM, Beer S, Kool J, Magistris MR, Kesselring J, Rösler KM (2004) Quantification of Uhthoff's phenomenon in multiple sclerosis: a magnetic stimulation study. Clin Neurophysiol 115:2493-2501

Hunter SK, Enoka RM (2003) Changes in muscle activation can prolong the endurance time of a submaximal isometric contraction in humans. J Appl Physiol 94:108-118

Kischka U, Fajfr R, Fellenberg T, Hess CW (1993) Facilitation of motor evoked potentials from magnetic brain stimulation in man: a comparative study of different target muscles. J Clin Neurophysiol 10:505-512

Kukulka CG, Moore MA, Russell AG (1986) Changes in human alphamotoneuron excitability during sustained maximum isometric contractions. Neurosci Lett 68:327-333 
Liepert J, Kotterba S, Tegenthoff M, Malin JP (1996) Central fatigue assessed by transcranial magnetic stimulation. Muscle Nerve 19:1429-1434

Magistris MR, Rösler KM, Truffert A, Myers JP (1998) Transcranial stimulation excites virtually all motor neurons supplying the target muscle. A demonstration and a method improving the study of motor evoked potentials. Brain 121((Pt 3)):437-450

Magistris MR, Rösler KM, Truffert A, Landis T, Hess CW (1999) A clinical study of motor evoked potentials using a triple stimulation technique. Brain 122(Pt 2):265-279

McKay WB, Tuel SM, Sherwood AM, Stokic DS, Dimitrijevic MR (1995) Focal depression of cortical excitability induced by fatiguing muscle contraction: a transcranial magnetic stimulation study. Exp Brain Res 105:276-282

McManis PG, Lambert EH, Daube JR (1986) The exercise test in periodic paralysis. Muscle Nerve 9:704-710

Mori S (1973) Discharge patterns of soleus motor units with associated changes in force exerted by foot during quiet stance in man. J Neurophysiol 36:458-471

Naka D, Mills KR (2000) Further evidence for corticomotor hyperexcitability in amyotrophic lateral sclerosis. Muscle Nerve 23:1044-1050

Pettorossi VE, Della Torre G, Bortolami R, Brunetti O (1999) The role of capsaicin-sensitive muscle afferents in fatigue-induced modulation of the monosynaptic reflex in the rat. J Physiol 515((Pt 2)):599-607

Pitcher JB, Miles TS (2002) Alterations in corticospinal excitability with imposed vs. voluntary fatigue in human hand muscles. J Appl Physiol 92:2131-2138

Ridding MC, Brouwer B, Miles TS, Pitcher JB, Thompson PD (2000) Changes in muscle responses to stimulation of the motor cortex induced by peripheral nerve stimulation in human subjects. Exp Brain Res 131:135-143

Rösler KM, Etter C, Truffert A, Hess CW, Magistris MR (1999) Rapid cortical motor output map changes assessed by the triple stimulation technique. Neuroreport 10:579-583

Rösler KM, Truffert A, Hess CW, Magistris MR (2000) Quantification of upper motor neuron loss in amyotrophic lateral sclerosis. Clin Neurophysiol 111:2208-2218

Rösler KM, Petrow E, Mathis J, Arányi Z, Hess CW, Magistris MR (2002) Effect of discharge desynchronization on the size of motor evoked potentials: an analysis. Clin Neurophysiol 113:1680-1687

Rothwell JC, Hallett M, Berardelli A, Eisen A, Rossini P, Paulus W (1999) Magnetic stimulation: motor evoked potentials. The
International Federation of Clinical Neurophysiology. Electroencephalogr Clin Neurophysiol Suppl 52:97-103

Samii A, Wassermann EM, Hallett M (1997) Post-exercise depression of motor evoked potentials as a function of exercise duration. Electroencephalogr Clin Neurophysiol 105:352-356

Stokes MJ, Edwards RH, Cooper RG (1989) Effect of low frequency fatigue on human muscle strength and fatigability during subsequent stimulated activity. Eur J Appl Physiol Occup Physiol 59:278-283

Taylor JL, Gandevia SC (2008) A comparison of central aspects of fatigue in submaximal and maximal voluntary contractions. J Appl Physiol 104:542-550

Taylor JL, Butler JE, Allen GM, Gandevia SC (1996) Changes in motor cortical excitability during human muscle fatigue. J Physiol 490:519-528

Taylor JL, Butler JE, Gandevia SC (1999) Altered responses of human elbow flexors to peripheral-nerve and cortical stimulation during a sustained maximal voluntary contraction. Exp Brain Res 127:108-115

Taylor JL, Petersen N, Butler JE, Gandevia SC (2000) Ischaemia after exercise does not reduce responses of human motoneurones to cortical or corticospinal tract stimulation. J Physiol 525:793-801

Uozumi T, Tsuji S, Murai Y (1991) Motor potentials evoked by magnetic stimulation of the motor cortex in normal subjects and patients with motor disorders. Electroencephalogr Clin Neurophysiol 81:251-256

Vagg R, Mogyoros I, Kiernan MC, Burke D (1998) Activitydependent hyperpolarization of human motor axons produced by natural activity. J Physiol 507((Pt 3)):919-925

Woods JJ, Furbush F, Bigland-Ritchie B (1987) Evidence for a fatigueinduced reflex inhibition of motoneuron firing rates. J Neurophysiol 58:125-137

Z'Graggen WJ, Humm AM, Durisch N, Magistris MR, Rösler KM (2005) Repetitive spinal motor neuron discharges following single transcranial magnetic stimuli: a quantitative study. Clin Neurophysiol 116:1628-1637

Zajac FE, Faden JS (1985) Relationship among recruitment order, axonal conduction velocity, and muscle-unit properties of typeidentified motor units in cat plantaris muscle. J Neurophysiol 53:1303-1322

Zytnicki D, Lafleur J, Horcholle-Bossavit G, Lamy F, Jami L (1990) Reduction of $\mathrm{Ib}$ autogenetic inhibition in motoneurons during contractions of an ankle extensor muscle in the cat. J Neurophysiol 64:1380-1389 